2020-11-04

\title{
A Structured Approach to Modifying Successful Heuristics
}

\author{
Martin, S
}

http://hdl.handle.net/10026.1/16551

10.5220/0010143702200225

Proceedings of the 12th International Joint Conference on Computational Intelligence SciTePress

All content in PEARL is protected by copyright law. Author manuscripts are made available in accordance with publisher policies. Please cite only the published version using the details provided on the item record or document. In the absence of an open licence (e.g. Creative Commons), permissions for further reuse of content should be sought from the publisher or author. 


\title{
A Structured Approach to Modifying Successful Heuristics
}

\author{
Simon P. Martin ${ }^{1} \mathbb{1}^{\mathrm{a}}$, Matthew J. Craven ${ }^{2} \mathbb{1}^{\mathrm{b}}$ and John R. Woodward ${ }^{3} \mathbb{C}^{\mathrm{c}}$ \\ ${ }^{1}$ Brook, Isle of Wight, PO30 4EU, U. K. \\ ${ }^{2}$ Centre for Mathematical Sciences, University of Plymouth, Drake Circus, Plymouth PL4 8AA, U. K. \\ ${ }^{3}$ Computer Science, Queen Mary University of London, Mile End Road, London E1 4FZ, U.K. \\ simon888martin@gmail.com,matthew.craven@plymouth.ac.uk,j.woodward@qmul.ac.uk
}

Keywords: Heuristic, Gadget, Approximation algorithm, Optimisation Problem.

\begin{abstract}
In some cases, heuristics may be transferred easily between different optimisation problems. This is the case if these problems are equivalent or dual (e.g., maximum clique and maximum independent set) or have similar objective functions. However, the link between problems can further be defined by the constraints that define them. This refining can be achieved by organising constraints into families and translating between them using gadgets. If two problems are in the same constraint family, the gadgets tell us how to map from one problem to another and which constraints are modified. This helps better understand a problem through its constraints and how best to use domain specific heuristics. In this position paper, we argue that this allows us to understand how to map between heuristics developed for one problem to heuristics for another problem, giving an example of how this might be achieved.
\end{abstract}

\section{Introduction}

Karp reductions show that if a problem can be reduced to a known NP-complete problem, then it too must be NP-complete (Karp, 1972). Such NPcomplete problems are commonly solved with metaheuristics (Krawiec et al., 2018), of which there many approaches (e.g., genetic algorithm, ant colony optimisation, particle swarm optimisation). The number of such approaches is growing dramatically.

(Sörensen, 2015) examined this plethora of metaheuristic approaches and identified the need for an underlying theory, observing that ostensibly distinct metaheuristics in fact share many key components. The author recommended that the design of metaheuristics be performed with regard to problem structure. This problem structure thus informs the design of components of metaheuristics, too. Seeds of such an approach were originally suggested by the work of (Cook, 1971), commonly referred to as Cook-Levin, who show that any problem in NP can be written as a collection of clauses in conjunctive normal form (SAT).

(Karp, 1972) showed that, supplementary to
Cook-Levin, there is a structure to NP-complete problems in general, finding reductions between his famous $21 \mathrm{NP}$-complete problems. These transitive reductions may be seen as fundamental relations between structures of each of the problems. Since then, there have been thousands of NP-complete problems produced and, since they are all NP-complete, there are fundamental reductions or relations between the structures of these problems. Combined with the work of Cook-Levin, this backs up the work of (Sörensen, 2015), suggesting that there is a definite structure between effective algorithms for NPcomplete problems. This also holds for NP-hard optimisation variants of these problems. Furthermore, this suggests that some extension of reductions between problems may be produced between those algorithms. This means that a fundamental view of metaheuristic frameworks is possible, reducing (or mapping) between metaheuristic components. Finally, (Trevisan et al., 2000) showed that by writing problems as linear programs, it is possible to construct optimal gadgets (in polynomial time) via reducing between the constraints (or families of constraints) of these linear programs.

\footnotetext{
a (iD https://orcid.org/0000-0003-1399-860X

b (iD) https://orcid.org/0000-0001-9522-6173

c (D) https://orcid.org/0000-0002-2093-8990
} 


\subsection{Motivation}

As there are many existing algorithms that are not designed for a specific problem (e.g., NSGA-II, SPEA2), and many existing NP-complete/hard problems, our approach aims to build a bridge between existing theoretical work done on NP-complete problems and existing empirical work done by the metaheuristics community.

It could be argued that a well-designed metaheuristic is designed for a problem. For example, the well-known Lin-Kernighan heuristic (Lin and Kernighan, 1973) proves effective for the Travelling Salesman Problem (TSP) since it explicitly treats the topology of the problem. As another example, hillclimbers are excellent at solving unimodal problems. Our aim is to allow metaheuristics which are proved to work well on one problem to be ported to other problems via gadget transformations. Thus we wish to make use of knowledge we already have about mapping between problems and use this knowledge to make new algorithms.

Further, we may interpret the following points from (Cook, 1971):

1. A problem in NP is a problem that is only solvable by a polynomial-time algorithm that runs on a Nondeterministic Turing Machine (NTM);

2. They show how to implement any algorithm on an NTM as a Boolean formula;

3. Since any problem can encoded as an Boolean formula, any algorithm can thus be encoded as an Boolean formula.

In other words, we do not concentrate on the problems in NP - instead, we should be focusing on the algorithms that solve them. We are motivated by this approach of focusing on the algorithms and using the mapping to exploit these algorithms.

We can exploit existing theory which effectively provides a mapping (refinement of problem constraints) between problems. We build on this by suggesting that an algorithm that performs well on one domain can then be applied to another domain via such a mapping between problems. Thus we wish to take a promising algorithm from one domain and enable it to be applied to a new domain (which hopefully will also be well performing), hoping to avoid the issue of the ever increasing number of metaheuristics, as mentioned by (Sörensen, 2015). Building on Trevisan (Trevisan et al., 2000), we show how a heuristic appropriate for a given problem can be modified to be used on another different problem.

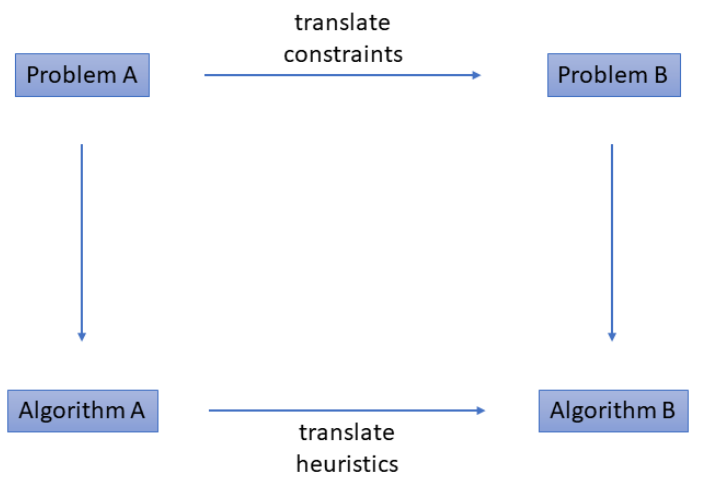

Figure 1: A diagrammatic explanation of our approach.

\subsection{Contribution of the Paper}

The contribution of this position paper is that the work of the aforementioned authors may be built upon to provide a method of producing mappings between heuristics. A visual explanation of our work is represented by Figure 1. Algorithms A and B denote algorithms that solve problems $\mathrm{A}$ and $\mathrm{B}$ respectively. The arrow between problems $\mathrm{A}$ and $\mathrm{B}$ denotes the translation of integer programming (IP) constraints from problem A to B via a gadget. That is, we translate modify or refine the constraints of problem A into those for problem B. Through an analogous approach to the gadget via judicious simulation of problem $B$ in Algorithm A, we create Algorithm B. Note that, of course, this makes no guarantees of the efficacy of the new algorithm (Algorithm B) on problem B. The vertical arrows denote that algorithm $\mathrm{A}$ is applied to problem A and algorithm B is applied to problem B. Our contribution is the concept and our further work will be the implementation.

Note that, in this position paper, we use the words 'algorithm' and 'heuristic' interchangeably. Also important to note that we do not make any guarantees of the efficiency of the new algorithm in this work; the implementation of such algorithms, based upon the ideas given in this work, is classified in the realms of further work.

This remainder of the paper is structured as follows. In Section 2 we introduce the technical background to our approach, examining constraints and their families, and then lead into gadgets. In Section 3 we detail our approach to constructing maps between algorithms by utilising existing gadgets defined between constraint families defining problems. We end the work in Section 4, giving a conclusion and an overview of further work. 


\section{Background}

In this section, we introduce the technical background to the work. Firstly, constraints that define problems and the families of constraints arising are examined, after which gadgets are briefly defined. Subsequently, an example of an existing gadget from Vertex Cover to Hamiltonian Circuit (both NP-complete problems) is given. Finally, these constraint families are partitioned into two types, the idea being gadgets to map between constraints in a particular type of constraint family are practical.

\subsection{Constraint Families and Gadgets}

In this paper, we write definitions of NP problems as IPs since it is straightforward to understand the constraints that define the problem. Constraint families are essentially a common language which allow us to show some problems are refinements of other problems. Further, (Trevisan et al., 2000) goes on to define constraint functions and constraint families, on which gadgets act, in terms of IPs. We adopt the common notation of $\mathbb{B}=\{0,1\}$. A constraint function is, by (Trevisan et al., 2000), simply a Boolean function $f: \mathbb{B}^{k} \rightarrow \mathbb{B}$. A constraint family is a set of constraint functions, and a constraint is then an assignment of a constraint function.

A gadget is an algorithm that transforms the constraints of one problem to another in polynomial time. Thus gadgets may be seen as methods for defining one problem in terms of another, only possible because of inherent constraint-wise similarities between those problems. Further, a gadget is, by (Trevisan et al., 2000, p. 2074), "a finite combinatorial structure which translates a given constraint of one optimization problem into a set of constraints of a second optimization problem." Thus, gadgets not only preserve (NP-)complexity, but structure also.

Historically, creating gadgets was, at the very least difficult and somewhat contrived, until the work of (Trevisan et al., 2000), using a linear programming (LP) implementation to find optimal gadgets in polynomial time. There is an extensive literature on gadgets (e.g., the works (Cai et al., 2012; Garey and Johnson, 1978; Papadimitriou and Yannakakis, 1988; Sipser, 2012; Skiena, 2008)). There is also a small amount of work on using gadgets to assist algorithms; e.g., the work of (Letchford and Vu, 2019) on using gadgets to generate cutting planes for algorithms on the Stable Set and Clique Partitioning problems.

\subsection{An Example of an Existing Gadget: Vertex Cover to Hamiltonian Circuit}

Reductions involve the modification of the constraints of one problem to encompass the constraints of another problem. We know that the Minimum Vertex Cover problem (i.e., finding the minimum set of vertices that touch every edge of a graph) is NP-complete via the famous reduction of its decision variant Vertex Cover from 3SAT (Skiena, 2008, pp. 333). We also recall that there is a reduction from Vertex Cover to Hamiltonian Circuit by manipulation of their respective IPs. Assume $G=(V, E, k)$ is an undirected graph, where $V$ is the set of vertices in the graph, $E \subseteq V \times V$ is the set of edges in the graph and $k$ is the size of the vertex cover.

For each vertex $v \in V$ we have a variable $x_{v}$. We interpret the variable as chosen to be included in a vertex cover if $x_{v}=1$, and otherwise $x_{v}=0$. The decision version of this problem is to return 1 if there is a vertex cover of size $k$, or 0 otherwise. The optimisation version of the problem is to minimise the number of vertices in the cover. As an IP, this is written as:

$$
\begin{aligned}
\min & \sum_{v \in V} x_{v}(\text { minimize total cost }) \\
& \text { subject to } \\
& x_{u}+x_{v} \geq 1 \text { for all } e=\{u, v\} \in E \\
& x_{v} \in\{0,1\} \text { for all } v \in V .
\end{aligned}
$$

The Hamiltonian Circuit problem is to decide whether, given a graph $G(V, E, k)$, there is a circuit of size $k$ of vertices, each with degree 2, where each vertex is visited only once. Here, $k$ is the required cycle size. Constraint (1) refers to covering every edge in the graph, whereas constraint (2) states that every vertex is either in or not in the cover.

Let $x_{i j} \in\{0,1\}$ be a variable representing an edge, with value 1 if the edge is part of the route connecting $v_{i}$ and $v_{j}$, and 0 otherwise. Let $c_{i j}$ be the distance (under some appropriate metric) between vertices $i$ and $j$. The following gives the well-known Miller-TuckerZemlin formulation of the TSP (Miller et al., 1960):

$$
\min \sum_{i=1}^{n} \sum_{j \neq i, j=1}^{n} c_{i j} x_{i j} \text { (minimise total distance) }
$$


subject to the following constraints:

$$
\begin{array}{ll}
x_{i j} \in\{0,1\} & i, j=1, \ldots, n \\
u_{i} \in \mathbb{Z} & i=2, \ldots, n \\
\sum_{i=1, i \neq j}^{n} x_{i j}=1 & j=1, \ldots, n \\
\sum_{j=1, j \neq i}^{n} x_{i j}=1 & i=1, \ldots, n \\
u_{i}-u_{j}+n x_{i j} \leq n-1 & 1<i \neq j \leq n . \\
0 \leq u_{i} \leq n-1 & 1<i \leq n
\end{array}
$$

Constraint (7) is the subtour constraint, designed to ensure that a tour does not break into at least two subtours (the vertices clearly cannot cross but edges, of course, may). Note that there are other formulations of the TSP that are possible (Langevin et al., 1990; Cheung, 2005; Sawik, 2016).

The well-known gadget of (Skiena, 2008) maps Vertex Cover to Hamiltonian Circuit. The gadget converts the instance of Vertex Cover into one of Hamiltonian Circuit. The process involves turning the undirected graph of the source problem into the digraph of the target problem. The edge gadget turns an undirected edge into a directed edge. The digraph of the target problem is then a chain of successive edge gadgets governed by the vertices originally connected together in the vertex cover. In terms of constraints, the edge gadget converts constraint (1) in the Vertex Cover IP, and maps it to constraints (5) and (6) of the Hamiltonian Circuit IP formulation.

We consider Vertex Cover and Hamiltonian Circuit to be part of the constraint family that we call allocation, where an algorithm allocates a vertex if it lies on the vertex cover or route.

\subsection{Constraint Families: Allocation and Partition}

The work of (Trevisan et al., 2000) discussed constraint families with respect to defining approximation algorithms. They used polynomial time reductions to define constraint families and showed how they could be used to help find new approximation algorithms. In this work, we study reductions using gadgets to show how they refine or redefine the constraints of one problem to encompass the constraints of another. In this way, they do not just preserve complexity from one problem to another, but problem structure (i.e., IP constraints) also. It is in this way gadgets can show how one problem is related to another in the context of constraint families.

Graphs are used to express combinatorial optimisation problems. The vertices simply represent "ob- jects", but the edges have a more complex role to play; edges have different interpretations depending on the problem. Let us compare three examples.

1. in Vertex Cover, an edge from one vertex to another vertex (assuming one vertex is in the cover set) tells us that the other vertex is covered;

2. in Graph Colouring, the edge tells us that each vertex (connected by the edge) must be a different colour; and

3. in Hamiltonian Circuit the edge tells us that this edge is part of the route.

The constraints tell us how to interpret and deal with the edges for each type of problem. In other words, vertices typically have a simple interpretation, i.e., "objects" in the problem, while edges are interpreted as the "relationship between objects", and give meaning to the different problems (i.e., expressed by the constraints). The meaning of a graph in terms of the problem being expressed originates from the interpretation of the edges, and not the vertices, and this is why focusing on the constraints is fundamentally important.

We have called the constraint family containing 3SAT, Vertex Cover and Hamiltonian Circuit allocation because the constraints cause the algorithm to allocate vertices with a certain property to a result set. The allocation constraint family essentially means using an edge to represent relations between elements of the source problem.

However, when this is not the case, this leads us to another constraint family we call partition. This involves using an edge to represent that two objects have no relation. In other words, we must partition a result set rather than allocate elements to one. The constraint family then represents those problems which involve partitioning or colouring sets, or the search for independent objects or subgraphs with given properties. Two examples of problems in this constraint family are 3-Colour, where we partition the vertices into three sets (each set corresponding to a different colour), and Subset Sum, where we partition into two sets (where one of the sets has a given target sum). Again, we can define this constraint family as we know there are reductions between problems in the constraint family (Garey and Johnson, 1979). In this way, the types of algorithms effective on problems from each type of constraint family may be analogously partitioned, and mapping between algorithms of a given type is possible because the key operations of the algorithm on each problem are essentially the same. The next section gives our proposed approach. 


\section{Our Proposed Approach}

\subsection{Introduction}

The field of metaheuristics has suffered in the past few years by an overwhelming number of unexplained algorithms. We believe to progress the field we need fewer, but better understood, algorithms. We propose a method of taking an algorithm designed with insights into one problem, and then making that algorithm applicable to a different problem type by using gadgets to map between the constraint classes of problems. This mapping is effectively a mapping between an existing algorithm and a new algorithm (see Figure 1).

\subsection{Steps in Approach}

The proposed approach involves the following steps.

1. Identify a successful heuristic on a specific domain (e.g., Lin-Kernighan on the TSP). We call this heuristic the source algorithm and the problem the source problem;

2. Either identify an existing gadget from the existing research literature, or design a new gadget which maps between the constraints of source problem and the target problem (e.g., a gadget mapping from TSP to Minimum Vertex Cover);

3. Then apply the existing source algorithm to the problem which has been translated from the target problem to the source problem.

This mapping process, in effect, gives us a new algorithm acting on a different problem to which it was intended by exploiting the mapping provided by the gadget. The gadget directly maps between the constraints of problems and indirectly maps between algorithms.

\subsection{An Example of the Approach}

We discuss a practical example of how this transformation might be achieved. We take a Vertex Cover problem such as one of the benchmark problems found at $(\mathrm{Xu}, 2014)$. We can then create an algorithm based on the gadget described in Section 2.2, and show how the Vertex Cover instance is transformed into a tour and solved accordingly. As such, this problem could be solved using a well-known TSP solver such as the Lin-Kernighan algorithm (Lin and Kernighan, 1973).

The gadget in Section 2.2 is an example of a polynomial time algorithm which maps one problem to another problem. It does not directly transform one algorithm into another. Rather, it shows how the constraints of one problem may be refined to the constraints of another. To do this we need to make a number of observations. A Vertex Cover graph has predefined edges and as such will have leaves that do not lead to other edges. It might also have highly interconnected subgraphs, which means there will be internal loops.

This means the gadget described shows how a solution to a Vertex Cover problem can also be a solution to Hamiltonian Circuit problem at the same time. If we look at constraint (1) of the Vertex Cover IP and constraints (5) and (6) of the Hamiltonian Circuit IP, we can see how the two Hamiltonian Circuit constraints are refinements of the Vertex Cover constraint. That is, an edge in a Vertex Cover can have at most two vertices in a cover set. As a brief example, take an edge from vertex $i$ to vertex $j$ in a Vertex Cover graph. This can be represented as a route from $i$ to $j$ if only one vertex of the edge is in the cover. If both vertices are in the cover we route from $i$ to $j$ and then $j$ to $i$.

From this observation we can suggest a method for solving Vertex Cover using an algorithm which is designed to solve Hamiltonian circuits. This might be efficacious as Hamiltonian Circuit and TSP problems have received a lot of attention by researchers and there are many algorithms that find good solutions to these types of problems. This is because constructive heuristics tell us something about the solution and the process by which we arrive at a solution. Let us define a $\{0,1\}$-adjacency matrix where the value 0 indicates the absence of an edge from $i$ to $j$, while the value 1 indicates the presence of an edge. We can modify a well-known algorithm for solving the vertex cover problem. Let $C=\emptyset$ be the cover set and let $E$ be the set of all edges in the graph $G$. Then while $E \neq \emptyset$ pick an arbitrary edge $(u, v)$ and put both vertices in the cover. Then remove all edges incident to those two vertices. Repeat until the set $E$ is empty. We can modify this algorithm to produce a Hamiltonian Circuit by noticing that the edges $(u, v)$ in the Vertex Cover algorithm can be seen as edges that are visited in both directions in any circuit, i.e., $(i, j)$ and $(j, i)$. The edges incident to these edges are of the type where they are visited only once in a Hamiltonian circuit. The next section concludes the paper.

\section{Conclusion}

This position paper has presented an approach for modification of a given heuristic acting on one prob- 
lem to be applicable on another problem, where the two problems are connected by a gadget. An existing gadget between Vertex Cover and Hamiltonian Circuit was detailed, after which constraint families were covered. These constraint families are vital to the "translatability" between IP formulations of problems. Subsequently, our proposed approach was introduced, using specifically the detailed gadget to refine the constraints from Vertex Cover to Hamiltonian Circuit. This then applies naturally to the optimisation versions of these two problems: Minimum Vertex Cover and the TSP. This clearly suggests further avenues of research with respect to implementation of the approach, experimentation and future generalisations.

What we are proposing here is a way to transfer between algorithms in order to bring about meaningful comparisons. Constraint classes and their respective gadgets allow us to translate from one problem to another and preserve underlying problem structure. It is this preserved problem structure we wish the new algorithm to be able to exploit. By representing problems as IPs, we can see which constraints need to be modified when transforming between problems. They also show us how to modify algorithms for solving one problem into algorithms to solve another. This observation is fundamental to our argument. The approach is useful since we can use it to modify existing algorithms intended for one domain, and therefore design "new" heuristics which are applied to a different domain (see Figure 1 for a graphical interpretation). This approach goes some way to addressing the concerns of (Sörensen, 2015). Our outlook, then, is a framework in which we can take algorithms designed for one problem, and meaningfully compare them with algorithms designed for another problem.

\section{Acknowledgements}

The authors should like to thank Queen Mary University of London and the University of Plymouth for their generous hospitality when writing this paper. Finally, the authors thank the anonymous referees for their suggestions which helped improve the work.

\section{REFERENCES}

Cai, J.-Y., Kowalczyk, M., and Williams, T. (2012). Gadgets and anti-gadgets leading to a complexity dichotomy. In Proceedings of the 3rd Innovations in Theoretical Computer Science Conference, ITCS '12, pages 452-467, New York, NY, USA. ACM.
Cheung, K. K. (2005). On Lovász-Schrijver liftand-project procedures on the Dantzig-FulkersonJohnson relaxation of the tsp. SIAM Journal on Optimization, 16(2):380-399.

Cook, S. A. (1971). The complexity of theorem-proving procedures. In Proceedings of the Third Annual ACM Symposium on Theory of Computing, pages 151-158. ACM.

Garey, M. R. and Johnson, D. S. (1978). "Strong" npcompleteness results: Motivation, examples, and implications. J. ACM, 25(3):499-508.

Garey, M. R. and Johnson, D. S. (1979). Computers and intractability: a guide to the theory of NP-completeness. W. H. Freeman \& Co., New York, NY, USA

Karp, R. M. (1972). Reducibility among combinatorial problems. Complexity of Computer Computations, pages 85-103.

Krawiec, K., Simons, C., Swan, J., and Woodward, J. (2018). Metaheuristic design patterns: new perspectives for larger-scale search architectures. In Handbook of Research on Emergent Applications of Optimization Algorithms, pages 1-36. IGI Global.

Langevin, A., Soumis, F., and Desrosiers, J. (1990). Classification of travelling salesman problem formulations. Operations Research Letters, 9(2):127-132.

Letchford, A. N. and Vu, A. N. (2019). Facets from gadgets. Mathematical Programming, pages 1-18.

Lin, S. and Kernighan, B. W. (1973). An effective heuristic algorithm for the traveling-salesman problem. Operations research, 21(2):498-516.

Miller, C. E., Tucker, A. W., and Zemlin, R. A. (1960). Integer programming formulation of traveling salesman problems. J. ACM, 7(4):326-329.

Papadimitriou, C. and Yannakakis, M. (1988). Optimization, approximation, and complexity classes. In Proceedings of the Twentieth Annual ACM Symposium on Theory of Computing, STOC '88, pages 229-234, New York, NY, USA. ACM.

Sawik, T. (2016). A note on the miller-tucker-zemlin model for the asymmetric traveling salesman problem. Bulletin of the Polish Academy of Sciences. Technical Sciences, 64(3).

Sipser, M. (2012). Introduction to the Theory of Computation. Cengage Learning.

Skiena, S. S. (2008). The algorithm design manual. Springer.

Sörensen, K. (2015). Metaheuristics - the metaphor exposed. International Transactions in Operational Research, 22(1):3-18.

Trevisan, L., Sorkin, G. B., Sudan, M., and Williamson, D. P. (2000). Gadgets, approximation, and linear programming. SIAM Journal on Computing, 29(6):20742097.

Xu, K. (2014). Benchmarks with hidden optimum solutions for graph problems. available at http://sites.nlsde.buaa.edu.cn/ kexu/benchmarks/ graph-benchmarks.htm. 